\title{
Scanning electron microscopy study of bone intracortical vessels using an injection and fractured surfaces technique
}

\author{
Ugo E. Pazzaglia $\cdot$ Terenzio Congiu • \\ Federico Ranchetti · Matteo Salari · \\ Carlo Dell'Orbo
}

Received: 18 February 2009/ Accepted: 29 May 2009/Published online: 7 July 2009

(C) Japanese Association of Anatomists 2009

\begin{abstract}
The intracortical canal/vessel systems of long bones are not yet completely understood in terms of their morphology and physiology, mainly because of the difficulty of injecting the small calibre vessels and cutting the calcified matrix. Here, we apply a novel method combining perfusion of the vessels and fracture of the cortical bone to enlighten the architecture of this system. The femurs of ten rabbits were perfused with a water-soluble dye (China ink) or alcoholic glycerol solution, and the fractured cortex specimens were then examined by scanning electron microscopy (SEM). The results document: (1) the fibrillar structure of the canal surfaces; (2) the perivascular environment with cellular components in different phases of incorporation within the bone matrix; (3) previously unreported filamentous structures on the outer surface of vessels, which could be interpreted as non-myelinic nerve fibres; (4) the inner organisation of the cutting cones. Although based exclusively on morphology, these observation have some relevance to increasing knowledge of bone circulation physiology in the cortical bone.
\end{abstract}

Keywords Intracortical vessels · Intracortical canals system · Cutting cone $\cdot$ Osteon · Bone remodelling · Bone vessels non-myelinated nerve fibres

U. E. Pazzaglia $(\varangle) \cdot$ F. Ranchetti · M. Salari Clinica Ortopedica dell'Università di Brescia, Spedali Civili di Brescia, 25123 Brescia, Italy e-mail: ortopedia2@spedalicivili.brescia.it

T. Congiu · C. Dell'Orbo

Dipartimeto di Istomorfologia dell'Università

dell'Insubria, 21100 Varese, Italy

\section{Introduction}

Current knowledge of the vascular supply of long bones is founded on a large number of studies in which a technique involving injection of the vascular tree was applied (Morgan 1959; Trias and Fery 1979; Rhinelander et al. 1979; Lopez-Curto et al. 1980; Bridgeman and Brookes 1996; Brookes and Revell 1998; Nelson et al. 1960). After perfusion with polymerising resins, it is possible to study the corrosion cast of bone vessel systems using scanning electron microscopy (SEM) (Irino et al. 1975; Draenert and Draenert 1980; Ohtani et al. 1982; Skawina et al. 1994), and to produce three-dimensional representations of the network of vessels that allow stereometric measurements to be made (Minnich et al. 1999; Minnich and Lametschwandtner 2006).

It is hugely difficult to obtain images of the intracortical system because the calibre of the vessels is small and they are contained inside a network of canals, which increases the resistance to perfusion; therefore, specific observations concerning this sector of the bone vessel system are few (Trias and Fery 1979; Nelson et al. 1960; Hert and Hladíková 1961; Lopez-Curto et al. 1980) and limited to histological preparations injected with water-soluble dyes.

To shed more light on the architecture of the intracortical vessel/canal system and the morphology of the perivascular space, we present here an original approach, which combines high-pressure perfusion of the vessels and fracture of the cortical bone. A water-soluble dye and alcoholic glycerol solution were used to compare artefacts produced by dehydration during processing. Direct SEM observation provided documentation of the canal and vessel surfaces in different phases of osteon organisation, such as the cutting cone advancement and the closure process of the osteon. 


\section{Materials and methods}

The study was carried out on the femurs of ten male, New Zealand white rabbits (Stefano Morini, S. Polo d'Enza, Reggio Emilia, Italy), weight between 3.0 and $3.5 \mathrm{~kg}$, about 8 months of age. Care and use of experimental animals was consistent with the procedures and regulations of the Italian Health Ministry.

To inject the vascular tree of the lower limbs, the rabbits were anaesthetized with ketamine cloridrate (Imagel) and xylazine (Rompum); the aorta and the cava vein were exposed through a midline abdominal incision and a $1.5 \mathrm{~mm}$ catheter was inserted in the aorta between the diaphragm and the origin of the arteries to the kidneys in a proximal-to-distal direction. The artery was then ligated tightly with two knots around the catheter and the rabbit was killed with a further overdose of the anaesthetic just before starting perfusion of the vascular tree.

A first group of six rabbits was injected with $300 \mathrm{ml}$ black China ink-water solution $(60 \%)$ with a hand syringe at a pressure of 150-200 mm mercury until the lower limbs were completely perfused (about $5 \mathrm{~s}$ ). The cava vein had been previously clamped with forceps because a high pressure in the extra-cortical vascular tree was necessary to balance the difference of the resistence to perfusion between the extra-cortical and intra-cortical vascular trees. With this procedure, complete perfusion of the intracortical sector vessels is obtained without any dilation of the injected vessels (Pazzaglia et al. 1997).

A second group of four rabbits was perfused in the same way with $300 \mathrm{ml}$ glycerol ethanol solution $(60 \%)$.

The skin of the limbs of the black China ink-injected animals was excised, and both femurs were dissected from soft tissues, and fixed in neutral formaline (10\%). The left femurs were decalcified in Osteosoft (Merck Sharp \& Dome, Rome, Italy) at $37^{\circ} \mathrm{C}$ for 2 months, and the mid-part of the diaphysis (about $10 \mathrm{~mm}$ long) was cut with a blade in a plane perpendicular to the major axis of the bone and the proximal and distal parts were discarded. The central cylinder of each femur was further sectioned: some specimens were observed unstained as full-thickness cortex (Pazzaglia et al. 2007), others were embedded in paraffin and transverse or longitudinal sections were stained with haematoxylin-eosine for standard histological observation.

After fixation in neutral formaline (10\%), the undecalcified right femurs were processed for SEM as follows:

Both right and left femurs of the four rabbits injected with glycerol were dissected from soft tissues and fixed in neutral formaline solution (10\%) for a week. Each of the eight mid-diaphyses and six of the black China ink-injected right femurs were cut with a hand saw into cylindrical segments of about $5 \mathrm{~mm}$. Each of these cylinders was split longitudinally into four pieces and each of these was fractured by notching one of the transversely cut surfaces with a chisel and opening the specimens with two flat nose pliers so to expose the fractured surface without direct handling (Fig. 1).

Specimens of each femur were divided into two groups and processed differently as follows:

The first group was submitted to osmic maceration. These specimens were washed in phosphate-buffered saline (PBS, pH 7.2) and then post-fixed in a solution of $1 \%$ osmium tetroxide and $1.25 \%$ potassium ferrocyanide for $2 \mathrm{~h}$. They were then washed in PBS, immersed in $0.1 \%$ osmium tetroxide in PBS for $72 \mathrm{~h}$ at room temperature and again washed in PBS.

The second group of specimens were cleaned for 2 weeks in a bath of hydrogen peroxide solution $(40 \%$ in water) to remove all soft tissues without mechanical manipulation, then washed in PBS. All specimens were dehydrated in ascending grades of ethanol, subjected to critical point drying in $\mathrm{CO}_{2}$, coated with $10 \mathrm{~nm}$ gold palladium in a vacuum sputter Emitech K550 (Edax, Mahwah, $\mathrm{NJ}$ ) and studied in the direct mode with a Philips XL 30 SEM-FEG scanning electron microscope (Philips, Eindhoven, The Netherlands).

\section{Results}

The fracture planes exposed either osteocytic lacunae, canalicula or vascular canals for a limited tract of their length. The vessels could be observed within their own canal when they had not been torn off by the mechanical damage of the fracture.

In China ink-injected specimens, vessel shrinkage formed an artefactual gap between the vascular structure and the inner canal surface (Fig. 2a). The outer surface of the vessel was smooth, with a loose mesh of fine collagen fibres, which in part stay adherent to the vessel wall and in part to the more densely packed collagen of the canal surface (Fig. 2b). Flattened cells could be observed at the interface between the vessel and the bone surface of the canal: these had cytoplasmic prolungaments at the
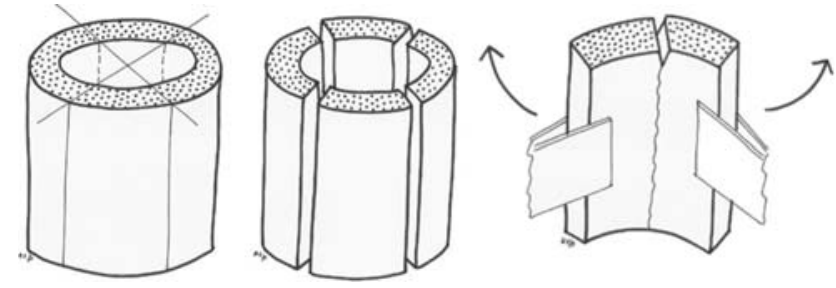

Fig. 1 Scheme showing the longitudinal cuts and the fracture technique to prepare the specimens for scanning electron microscopy (SEM) observation 


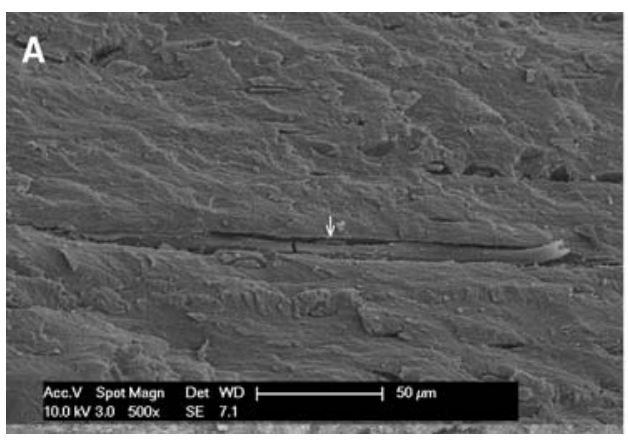

Fig. 2 a Scanning electron micrograph (SEM) of the fractured surface of black China ink-injected specimen $(\times 500)$. The fracture plane runs along an intracortical canal exposing a wide tract of the vessel. Shrinkage has detached the vessel from the surface of the canal. An osteoblast/osteocyte is evident between the vessel and the canal wall (arrow). b SEM of the fractured surface of black China

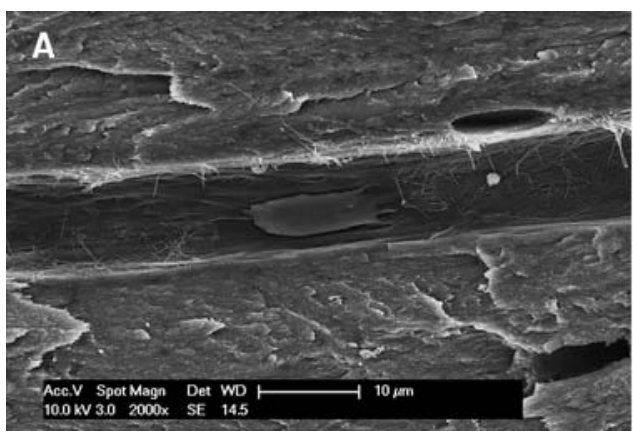

Fig. 3 a SEM of the fractured surface of a black China ink-injected specimen $(\times 2,000)$. The well structured collagen bundles of the canal surface and an osteoblast showing a flat surface toward the vessel can be seen, while cellular prolungaments enter inside the canalicula of the matrix at the periphery of the cell. Fine collagen fibres disorderly arranged are present on the canal surface. b SEM of the fractured surface of a specimen treated with osmium maceration $(\times 3,500)$. The

periphery and on the surface facing the bone. On the contrary, they appeared smooth on the surface facing the vessel (Fig. 3a). Occasionally, their osteocytic lacunae could be observed in the process of becoming surrounded by the matrix (Fig. 3b). The canal surface had an ordered interwoven texture of collagen fibres, which could be better seen after osmium maceration treatment (Fig. 3b).

The fracture plane of bones injected with glycerol alcoholic solution did not show any preference for the vascular canals, therefore the tracts exposed were shorter than in water-soluble dye-injected bones, and the vessels could be observed only through osteocytic lacunae or other bone defects exposed by the fracture. The shape of the latter vessels was better preserved, and they appeared as straight tubes with a regular circumference; the gap between the bone and the vessel was less evident. Thin and loose collagen fibres were in continuous with the fibrillar structure of the bone matrix. Surface osteoblasts presented an hemi-lacuna with typical canalicula holes (Fig. 4a). At

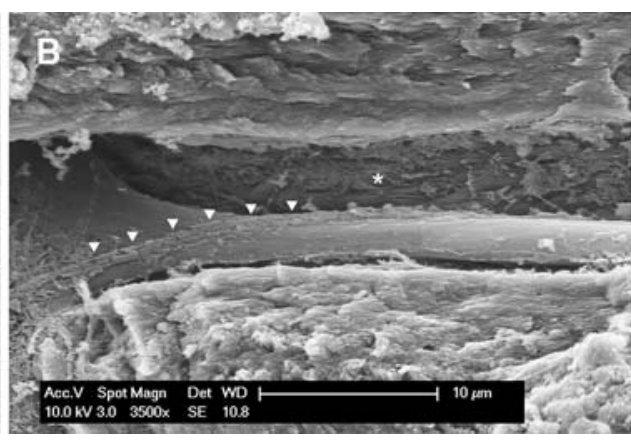

ink-injected specimen $(\times 3,500)$. Bifurcation of a vessel inside its own canal: the canal surface (asterisk) can be seen in the background covered by organic deposits. Filaments, distinct from the basal membrane, adhere to the outer surface of the vessel (inverted triangles); these have been interpreted as non-myelinated nerve fibres

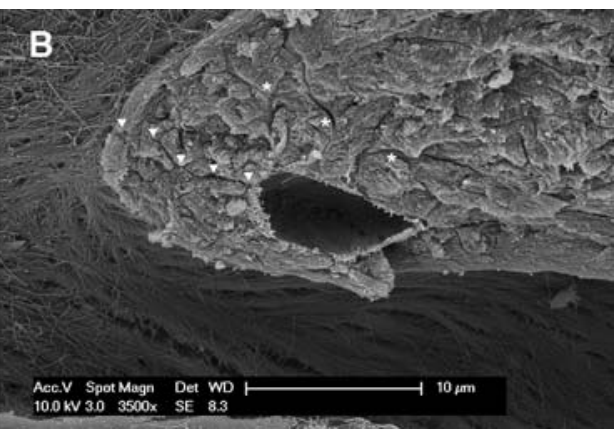

partial removal of organic deposits has evidenced the interwoven texture of collagen bundles of the canal surface. The inner surface of the osteocytic lacuna shows a similar collagen texture, but with thinner bundles. Many canalicula (asterisks) irradiate from the osteocytic lacuna, connecting the perivascular space of the canal with the osteocytic lacuna (inverted triangles)

higher magnification, the vessel wall was represented by a layer of collagen fibres outside the basal membrane, with the same interwoven organisation as seen on the canal surface (Fig. 4b).

Tears in vessels produced occasionally during specimen processing showed the smooth endothelial luminal surface, revealing the fine collagen fibres texture of the basal membrane and its connections with the most superficial fibre layer of the canal. Typically, blood cells were present inside the lumen (Fig. 5).

Elongated and interconnecting filaments, some flattened like a ribbon, and between 0.1 and $1 \mu \mathrm{m}$ in size, were observed either on the canal wall (Fig. 6a) or on the external surface of the vessel (Fig. 6b) when the fracture plane divided the two structures. Therefore they were positioned between the basal membrane of the vessel and the interwoven collagen bundles of the canal wall. Short and larger circumferential processes were occasionally observed around the vessel (Fig. 6b). 


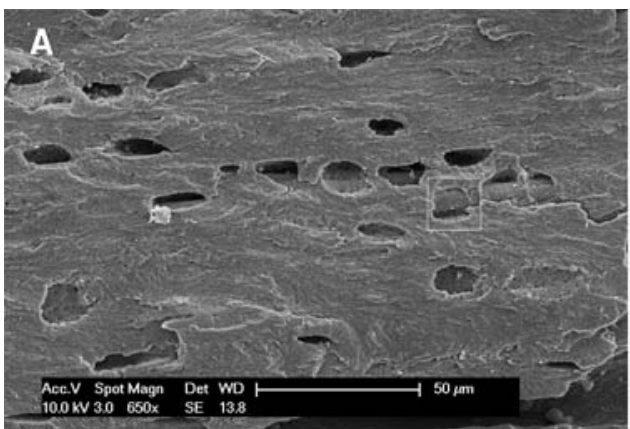

Fig. 4 a SEM of the fractured surface of a glycerol-injected specimen $(\times 650)$. The fracture plane does not pass along any intracortical canal and the vessels can be observed through osteocytic lacunae or other bone defects. The gap between the vessel and the canal surface is much less evident than in water-soluble dye injected

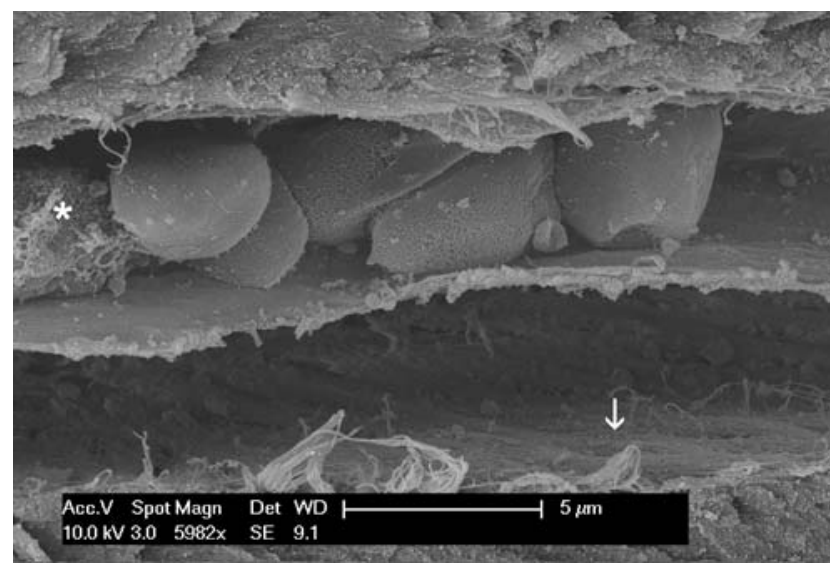

Fig. 5 SEM of the fractured surface of a glycerol-injected specimen $(\times 5,982)$. Torn vessel showing red cells and a polymorphonuclear neutrophil (asterisk) inside the lumen. The top half of the vessel remained attached to the canal surface, while in the bottom half the basal membrane has been detached from the canal wall and the interwoven texture can be seen (arrow)

The cutting cones exposed by the fracture plane revealed large and flattened osteoclasts on the advancing front; the vessels of the loop were not evident because they were covered by a large number of cells of fusated shape. There was a space with lower cellular density between the osteoclasts and the vascular-connective structure in the centre of the cutting cone. Occasionally, transverse canals (Volkmann) were intercepted by the advancing cutting cone, forming a new connection in the canal network (Fig. 7). In fractured surfaces where the cells had been removed, resorption pits were evident on the front of the cone: the tips of truncated collagen fibres were exposed on the surface. At the back of the resorbing zone, osteoblasts spread their short prolungaments on the surface.

The whole vascular loop of the cutting cone could be observed in full-thickness specimens of the cortex by light microscopy (Fig. 8a), and never presented with a length of

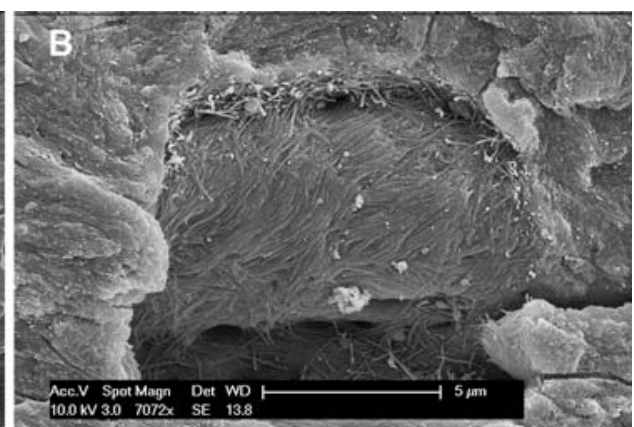

vessels. b SEM of the fractured surface of a glycerol-injected specimen $(\times 7,072)$. The figure shows an enlargement of the box in $\mathbf{a}$, showing the interwoven collagen bundles that cover the vessel; these pertain to the most superficial collagen layer present in the canal, and form a scaffold that contains the vessel

more than $1,000-1,200 \mu \mathrm{m}$; the usual aspect of the structured osteon was that of a central canal with a single capillary-like vessel (Fig. 8b), suggestive of a forced direction of blood flow in the loop (Fig. 8c).

\section{Discussion}

The combination of vessel perfusion and cortical bone fracture allowed the morphology of the intracortical vessels and of the perivascular space, which are not easily accessible to microscopic examination, to be examined by SEM. Mechanical dissociation of concentrical lamellae has been used so far to study osteon organisation (Frasca et al. 1977, 1981); however, in this case, particular problems originate from the presence of an interface between the rigid and hard scaffold of the calcified matrix and the soft tissue of the vessel, which introduces severe artefacts into the structures under observation. The mechanical force applied to fracture the cortex follows the lines of least resistance drawn by the intracortical canal networks; therefore, the plane of separation is irregular and presents only an approximate correspondence with the spatial dimensions of the planes of the anatomical structures under study (coronal, sagittal, horizontal), but it has the advantage of exposing a tract of the intracortical canal or a sector of the network wider than with any other technique where the cut section is rigidly fixed by the cutting device. It is not possible to guide the fracture through a pre-determined cross-section of the canal; however, a sufficiently wide exposure of the inner surface can usually be obtained by tilting the specimen. Cells and soft tissue structures present within the canal can be damaged, completely wrenched off, or only displaced from their original position; therefore, not all the canals exposed are suitable for study. However, some of the mechanical artefacts, such as tears of the vessel walls, can be used to reveal details of their structure. 


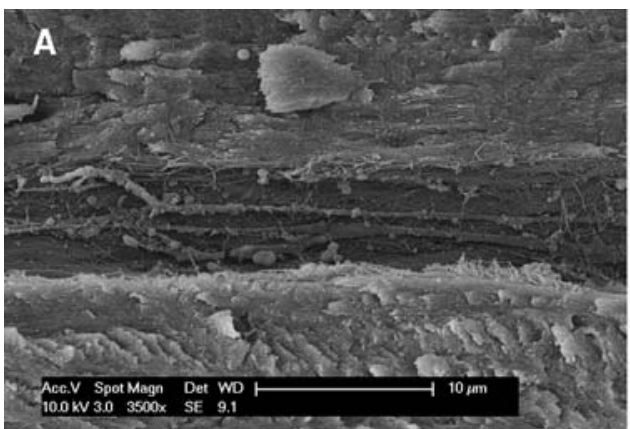

Fig. 6 a SEM of the fractured surface of a black China ink-injected specimen $(\times 3,500)$. Filaments and ribbons, distinct from collagen, have detached from the vessel surface and remain adhered to the canal wall; these have been interpreted as non-myelinated nerve fibres. b SEM of the fractured surface of a glycerol-injected specimen

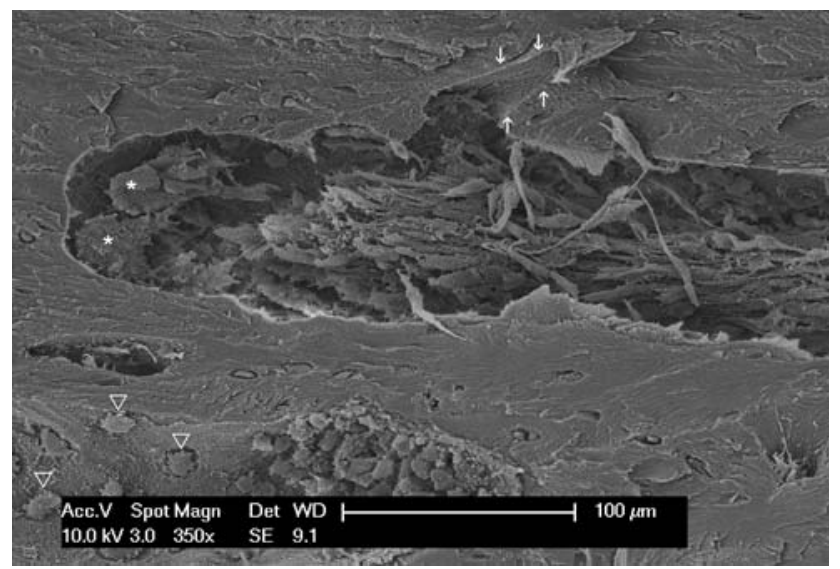

Fig. 7 SEM of the fractured surface of a black China ink-injected specimen $(\times 350)$. The fracture plane opened a cutting cone. Osteoclasts (asterisk) are evident on the advancing front. Behind, a cluster of fusated cells surrounds and completely covers the vascular loop. An intersection of the cutting cone with a pre-existing canal is evident on the upper border (arrows). In the lower-left corner a second cutting cone has been opened and the vascular loop detached; the flattened cells with prolungaments are osteoblasts (open inverted triangles)

A further artefact is shrinkage of soft tissues. In vessels injected with water-soluble dye, the latter produce a wide gap between the inner surface of the central canal and the wall of the vessel. Shrinkage resulted much lower in vessels injected with the alcoholic solution of glycerol, where the interface between the vessel basal membrane and the collagen bundles of the canal wall appeared almost virtual, with only a few, thin fibres spanning from one layer to the other. Shrinkage also influenced the fracture plane, because exposure of glycerol-injected vessels occurred only through osteocyte lacunae or other bone defects rather than along intracortical canals. These aspects of the glycerol-injected vessels revealed that the thin basal membrane closely fits the bone matrix scaffold; this observation has

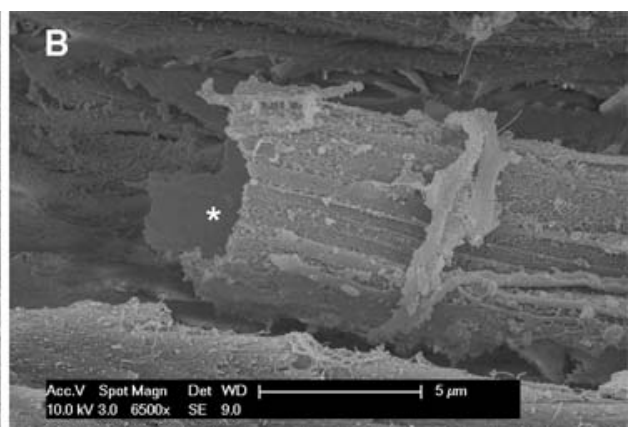

$(\times 6,500)$. Torn vessel showing the endothelial luminal surface (asterisk). The thin, longitudinal filaments on the surface of the vessel have been interpreted as non-myelinated nerve fibres, and the larger circumferential ribbon as a secondary pericytic process

also functional relevance, because it means that there is no possibility of dilation to increase the flow.

A view of the bone vascular network injected with resin can also be obtained using a corrosion cast technique (Irino et al. 1975; Ohtani et al. 1982; Skawina et al. 1994); however, most such observations concern marrow and periosteal vessels, while intracortical capillaries were seldom evidenced because of the high viscosity of the resin and difficulty of perfusion.

The artefactual gap in water-soluble dye-injected bones allowed a direct view of the cells present on the outer surface of the vessel, and of their relationship with the scaffold; in particular a clear polarisation was observed, with many cellular prolungaments toward the bone and a smooth cellular membrane on the side facing the vessel. Different degrees of encapsulation of these cells within the bone matrix were observed, supporting the view that they are osteoblasts in the process of becoming osteocytes.

The elongated filaments or ribbons on the vessel outer surface or adhering to the inner canal surface have, to the best of our knowledge, never been described previously with SEM. They are completely distinct from the collagen of the canal and the basal membrane of the vessel. Pericytes have been documented around the pre-capillary, capillary and post-capillary network in several organs and tissues (Higuchi et al. 2000); in particular capillary pericyte processes have been distinguished in longitudinal and short circumferential (Shimada et al. 1992): the latter were observed around the intracortical capillaries, documenting the presence of pericytes inside the bone network; however, the straight and regular filaments observed here were longer and thinner than pericyte longitudinal processes. In a transmission electron microscopy study, Cooper et al. (1966) documented nerve fibres around intracortical vessels; there is a good topographic correspondence between their ultrastructural findings and the surface filaments, therefore our interpretation is that they could correspond to 


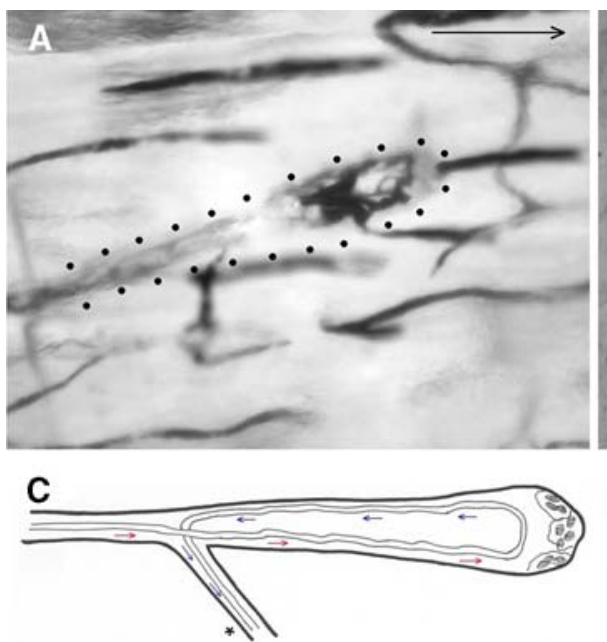

Fig. 8 a Full-thickness cortex injected with black China ink $(\times 400)$. A cutting cone vascular loop was injected with the contrast dye (sequence of points). The advancing front is indicated by the arrow in the top right corner and corresponds to the larger tract of the cone, which contains the loop. The cone size is progressively reduced by the closure process of the osteon. b Haematoxylin-eosine/black China ink staining $(\times 1,000)$. Osteon in an advanced phase of closure with a

non-myelinated nerve fibres of the small intracortical vessels.

The reported observations are based on morphology; however, they can have some relevance to our understanding of bone circulation physiology (Brookes et al. 1961; Brookes and Revell 1998; Gross et al. 1979; Cohen and Harris 1958). It is doubtful that these fibres could function to regulate blood flow by acting directly on the calibre of the small intracortical vessel, whose wall is formed only by the basal membrane and the endothelial cells. Therefore, a more likely hypothesis would be that only sensitive signals are conducted through these fibres.

The study of blood flow dynamics in bone cortex has been limited by the impossibility of using direct methods such as electromagnetic flow-meters or Doppler ultrasound, and most measurements have been made using radionuclides or microspheres (Tothill 1984; Michelsen 1967; Lunde and Michelsen 1970; Gross et al. 1979; Brinker et al. 1990; Willans and McCarthy 1991). The general view is that of an intracortical blood flow controlled by a pressure gradient between the marrow and periosteal arterioles and the drainage venous system (both extra-cortical). The structural aspects evidenced by SEM do not contradict the results of these haemodynamics studies.

The only vessels with contractile capacity within the canal system are the arterioles of the cutting cone vascular loop (Shenk and Willenegger 1964; Pazzaglia et al. 2008). With the technique applied here, it was not possible to highlight this loop because the central connective-vascular trellis was covered by a sheet of fusated cells that adhered

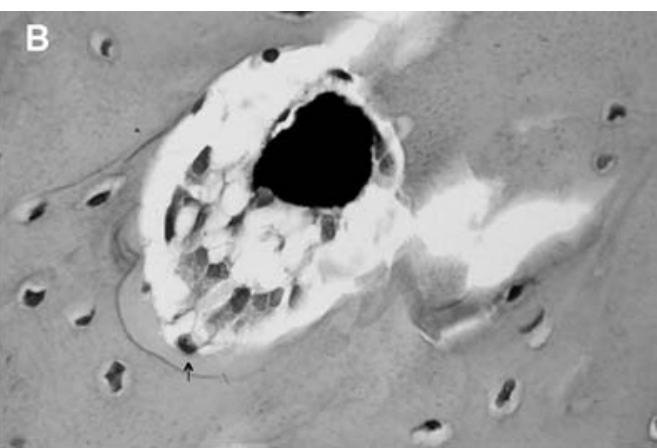

single capillary-like central vessel. Osteoblasts in the bottom of the canal are starting to form a bone lamella (arrow). c Scheme illustrating the hypothesis of the vascular flow inside the cutting cone loop: because, as a rule, a single capillary-like vessel is present in the central canal of the osteon, this suggests how the return flow could occur

to the surface of the weaving vessels; however, its spatial pattern could have been well evidenced by light-microscope observations of the full thickness cortex. On SEM, the cutting cone showed the clear spatial disposition of the different cell types: osteoclasts on the front of advancement, osteoblasts behind and lining the bony tunnel whose circumference was progressively reduced as the distance from the cutting head increased, and the connective-vascular axis in a central position. At a distance of about $700 \mu \mathrm{m}$, the size of the canal was reduced in such a way that there was space for only a single, capillary-like vessel. Considering the blood flow in the loop, it can be hypothesised that the return flow can occur only through the connections established by the advancing cutting head, with the lateral branches of the canals network that have been opened by the advancing cutting cone. The morphological data presented in this study are not in conflict with the hypothesis that blood flow in the loop is in the same direction as the advancement of the cutting cone. On the contrary, it is not possible to establish flow direction in other branches of the network because it depends on outside factors and can be assumed to be variable in relation to changes in the pressure gradient between the afferent of the system (branches of the inner marrow, of the metaphyseal and periosteal arteries) and drainage by the corresponding veins.

Acknowledgments The study was carried out with a scanning electron microscope of "Centre Great Instruments" of Insubria University, and was supported by research funds of Brescia University. 


\section{References}

Bridgeman G, Brookes M (1996) Blood supply to the human femoral diaphysis in youth and senescence. J Anat 188:611-621

Brinker NR, Lippton HL, Cook SD, Hyman AL (1990) Pharmacological regulation of the circulation of bone. J Bone J Surg 72A:964-975

Brookes M, Revell WJ (1998) Blood supply of bone. Scientific aspects. Springer, London

Brookes M, Elkin AC, Harrison RG, Heald CB (1961) A new concept of capillary circulation in bone cortex some clinical applications. Lancet i:1078-1081

Cohen J, Harris WH (1958) The three-dimensional anatomy of Haversian systems. J Bone Joint Surg (Am) 40A:419-434

Cooper RR, Milgram JW, Robinson RA (1966) Morphology of the osteon: an electron microscopic study. J Bone J Surg 48A:12391271

Draenert K, Draenert Y (1980) The vascular system of bone marrow. Scan Electron Microsc 4:113-122

Frasca P, Harper RA, Katz JL (1977) Collagen fiber orientations in human secondary osteons. Acta Anat 98:1-13

Frasca P, Harper RA, Katz JL (1981) Scanning electron microscopy studies of collagen, mineral and ground substance in human cortical bone. Scan Electron Micros III:339-346

Gross MP, Heistad DD, Marcus NL (1979) Neurohumoral regulation to blood flow to bones and marrow. Am J Physiol 237H:440-448

Hert J, Hladíková J (1961) Die Gefässversorgung des Haversschen Knoches. Acta Anat 45:344-361

Higuchi K, Hashizume H, Aizawa Y, Ushiki T (2000) Scanning electron microscopic study of the vascular smooth muscle cells and pericytes in the rat hearth. Arch Histol Cytol 63:115-126

Irino S, Ono T, Watanabe K, Toyota K, Uno J, Tanasugi n, Murakami $\mathrm{T}$ (1975) Scanning electron microscopic studies on microvascular architecture sinus wall and transmural passage of bone cells in the bone marrow by a new method of injection replica and non coated specimens. In: Proceedings of the 8th Annual Scanning Electron Microscope Symposium III Research Institute. Chicago, Illinois, April 1975

Lopez-Curto JA, Bassingthwaighte JB, Kelly PJ (1980) Anatomy of the microvasculature of the tibial diaphysis of the adult dog. J Bone Joint Surg 62A:1362-1369

Lunde PKM, Michelsen K (1970) Determination of cortical blood flow in rabbit femur by radioactive microspheres. Acta Physiol Scand 80:39-44

Michelsen K (1967) Pressure relationships in the bone marrow vascular bed. Acta Physiol Scand 71:16-29
Minnich B, Leeb H, Bernoider EWN, Lametschwandtner A (1999) Three-dimensional morphometry in scanning electron microscopy: a technique for accurate dimensional and angular measurements of microstructures using stereopaired digitized images and digital image analysis. J Microsc 195:23-33

Minnich B, Lametshwandtner A (2006) Lengths measurements in microvascular corrosion castings: two dimensional versus threedimensional morphometry. Scanning 22:173-177

Morgan JD (1959) Blood supply of growing rabbit's tibia. J Bone Joint Surg 41B:185-203

Nelson GE Jr, Kelly PJ, Peterson LFA, James JM (1960) Blood supply of the human tibia. J Bone Joint Surg 42A:625-636

Ohtani O, Gannon B, Ohtsuka A, Murakami T (1982) The microvasculature of bone and especially of bone marrow as studied by scanning electron microscopy of vascular cast. A review. Scan Electron Microsc 23:427-434

Pazzaglia UE, Andrini L, Di Nucci A (1997) The reaction to nailing or cementino of the femur in rats. A microangiographic and fluorescence study. Int Orthop 21:267-273

Pazzaglia UE, Bonaspetti G, Rodella LF, Ranchetti F, Azzola F (2007) Design morphometry and development of the secondary osteonal system in the femural shaft of the rabbit. $J$ Anat 211:303-312

Pazzaglia UE, Bonaspetti G, Ranchetti F, Bettinsoli PF (2008) A model of the intracortical vascular system of long bones and of its development. Experimental study in rabbit femur and tibia. J Anat 213:183-193

Rhinelander FW, Stewart CL, Wilson JW (1979) Bone vascular supply. In: Simmons DL, Kunin AS (eds) Skeletal Research. Academic, New York

Shenk R, Willenegger H (1964) Zur Histologie der primären Knochenheilung. Langenbecks Arch Chir 308:440-452

Shimada T, Kitamura H, Nakamura M (1992) Three-dimensional architecture of pericytes with special reference to their topographical relationship to microvascular beds. Arch Histol Cytol 55(suppl):77-85

Skawina A, Litwin JA, Gorczyca J, Miodonski AJ (1994) The vascular system of human fetal long bones: a scanning electron microscope study of corrosion casts. J Anat 185:369-376

Tothill P (1984) Bone blood flow measurement. J Biomed Eng 6:251256

Trias A, Fery A (1979) Cortical circulation of long bones. J Bone Joint Surg 61A:1052-1059

Willans SM, McCarthy ID (1991) Heterogeneity of blood flow in tibial cortical bone: an experimental investigation using microspheres. J Orthop Res 9:168-173 